\title{
Antropología
}

\section{Influencia generacional en el proceso de pérdida de las habilidades cuidadoras en la familia ante urgencias leves. Estudio piloto}

Generational influence on the process of loss of family caregivers skills to minor emergencies. Pilot study

Geracional influência sobre o processo de perda das habilidades de cuidadores familiares de pequenas emergências. Estudo piloto

Sonia Herrera Justicia ${ }^{1}$, Manuel Amezcua ${ }^{2}$

\begin{abstract}
${ }^{1}$ Doctoranda por la Universidad de Granada, Enfermera. Fundación Index, Granada, España ${ }^{2}$ Doctor por la Universidad de Alicante. Profesor titular del Centro Universitario de Enfermería San Juan de Dios, Universidad de Sevilla, España
\end{abstract}

Cómo citar este artículo en edición digital: Herrera Justicia, S. \& Amezcua, M. (2018). Influencia generacional en el proceso de pérdida de las habilidades cuidadoras en la familia ante urgencias leves. Estudio piloto. Cultura de los Cuidados (Edición digital), 22(52). Recuperado de http://dx.doi.org/10.14198/cuid.2018.52.11

Correspondencia: Calle Isleta de San Felipe, 8, 18001, Granada (España)

Correo electrónico: soniaherrera@correo.ugr.es Recibido: 16/04/2018; Aceptado: 20/06/2018

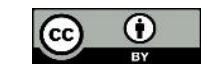

\begin{abstract}
Objective: To know the awareness of loss of skills caregivers in the family to minor emergencies related to the generational change.
\end{abstract}

Methodology: The study was addressed from an ethnographic qualitative, descriptive design. They interviewed six informants belonging to two families in which there were at least three generations related to each other. The data was collected through a semi-structured interview. An analysis of the discourse of semantic type was made from a double plane: intracases and intercases.

Main results: Most of the samples go to emergency departments by pathology of banal kind of motivations that lead to frequent this service are: go at own discretion, guided by the advice of others and derived from its health center. The entire sample is capable of telling some sort 
of remedy for the problem of health consulted, although few are still making use of these remedies.

\section{Conclusions: With regarding} intergenerational knowledge, exists in both sequences although information with the advance of generations is lost. Finally, the totalities of the interviewees are aware of the process of loss of cultural knowledge, among the reasons stand out: the influence of the modern pharmacy, passivity by the population, lack of confidence in these remedies and the influence of Oficial Medicine. The study shows the relevance of the revitalization of knowledge and caregiving skills in the family in order to rationalize the frequency of emergencies.

Key Words: Transcultural Nursing, cultural evolution, family, emergencies, complementary therapies.

\section{RESUMO}

Objetivo: Sei que a consciência da perda dos cuidadores habilidades da família para pequenas emergências relacionadas com a mudança geracional.

Método: $\mathrm{O}$ estudo dirigido de um design descritivo qualitativo etnográfico. Foram entrevistados seis informantes pertencentes a dois famílias em que havia pelo menos três gerações relacionadas entre si. Os dados foram coletados através de uma entrevista semi-estruturada. Se realize um análisis do discurso de tipo semântico de um duplo plano: intracasos e intercasos.

Resultados: A amostra vai para emergências, patologia banal, motivada pelo seu critério, pressionado por membros da família e derivado de seu centro de saúde. Eles foram capazes de dizer algum remédio para o problema de saúde que levou-os a visitar a sala de emergência, mesmo se eles não usá-los. Em relação ao conhecimento intergeracional, verificou-se que você perca informações com o avanço das gerações. Os entrevistados estão cientes do processo de perda de conhecimentos culturais, as razões incluem: influência da farmácia moderna, passividade da população, falta de confiança nestes remédios e influência da Medicina Oficial.

Conclusão: $\mathrm{O}$ estudo mostra a relevância da revitalização do conhecimento e das habilidades de cuidar na família, a fim de racionalizar a freqüência de emergências.

Palavras chave: PC Enfermagem Transcultural, evolução cultural, familia, emergências, terapias complementares.

\section{RESUMEN}

Objetivo: Conocer la conciencia de pérdida de las habilidades cuidadoras en la familia ante urgencias leves relacionada con el cambio generacional.

Metodología: El estudio se abordó desde un diseño cualitativo, descriptivo con enfoque etnográfico. Se entrevistaron a seis informantes pertenecientes a dos familias en las que existían al menos tres generaciones relacionadas entre sí. Los datos se recogieron a través de entrevista semiestructurada. Se realizó un análisis del discurso de tipo semántico desde un doble plano: intracasos e intercasos.

Resultados: La muestra acude a urgencias por patología banal, motivadas por decisión propia, presionados por familiares $y$ derivados desde su centro de salud. Fueron capaces de narrar algún remedio para el problema de salud que les llevó a visitar urgencias, aunque no hacen uso de los mismos. Con respecto al conocimiento intergeneracional, se comprobó que se perdía información con el avance de las generaciones. Los entrevistados son conscientes del proceso de pérdida de los saberes culturales, entre los motivos destacan: influencia de la farmacia moderna, 
pasividad por parte de la población, falta de confianza en estos remedios e influencia de la Medicina Oficial.

Conclusiones: El estudio muestra la pertinencia de la revitalización de saberes y habilidades cuidadoras en la familia de cara a racionalizar la frecuentación de urgencias.

Palabras clave: Enfermería Transcultural, evolución cultural, familia, urgencias médicas, terapias complementarias.

\section{INTRODUCCIÓN}

Los profesionales de salud son formados mayoritariamente bajo un modelo de salud dominante, que se denomina alopático, que pone especial énfasis en el paradigma científico positivista como método unitario de conocimiento y a menudo se muestra intolerante con otros saberes. Sin embargo, el ser humano siempre ha buscado distintas formas de sanar sus enfermedades en función básicamente de la explicación que le dé a la misma, que tiene la mayoría de las veces connotaciones culturales. Una vez que un individuo analiza el porqué de su enfermedad, pasa a solucionar su problema dentro del abanico de posibilidades que ofrece el sistema de salud (Echevarría, 2008). Según Glittenberg tenemos que eliminar la idea tradicional de asistencia sanitaria, ya que asocia la salud con lo que ocurre dentro de un hospital o con la limitada definición de una enfermedad concreta. Debemos ir más allá y ser plenamente conscientes del papel que representamos como impulsores de la salud y bienestar social de todos los ciudadanos, generando espacios de diálogo interactivo, creando nuevas formas de cuidado, donde se incentive a las personas a utilizar sus capacidades de curación y cuidados de acuerdo con sus propios contextos culturales; reafirmando el papel del sujeto en salud, donde "curar es papel de todos" (Glittenberg, 2010; Trinidade et al., 2013).

$\mathrm{Si}$ nos centramos en los Servicios de Urgencias en España vemos que suponen un volumen importante en las prestaciones que el Sistema Nacional de Salud pone a disposición de la población y que paradójicamente aunque el número de urgencias aumente, el $70 \%$ de las consultas son consideradas procesos leves o banales con la consecuencia de la masificación de este servicio (Ministerio de Sanidad, 2015; Miró, 2010 Palanca et al., 2010). Diversos estudios lo relacionan con los componentes sociológicos y culturales (la denominada "cultura de la inmediatez") que el sistema ha ido inculcando a la población y que ha provocado que las habilidades cuidadoras en la familia que durante siglos han sido el primer tratamiento que los mayores daban a sus dolencias, se sustituyan por el "acelerado consumismo sanitario" (González, 2010; Güell, 2008).

Este trabajo se plantea desde el convencimiento de que fomentando las habilidades cuidadoras en el seno familiar, se promovería un uso más racional de los servicios de urgencias. La Enfermería transcultural es el ideal a alcanzar en una sociedad como la actual. El principal mandato de la teoría de Madeleine Leininger es entender las tradiciones en salud y respetarlas más que desecharlas, ya que estas son parte de la vida de los pueblos.

El objetivo de este estudio es conocer la conciencia de pérdida de las habilidades cuidadoras en la familia ante urgencias leves relacionada con el cambio generacional.

\section{METODOLOGÍA}

El estudio se abordó desde un diseño de Investigación Cualitativa descriptivo con enfoque etnográfico. El estudio se realizó en 
Jaén capital. Criterios de inclusión: pertenecer a un estrato de una secuencia familiar de tres generaciones que tenía constituida su propia familia nuclear. La selección de sujetos se realizó mediante el muestreo por conveniencia.

Las categorías temáticas del estudio fueron: motivo por el cual acuden a los servicios de urgencia, existencia de conocimiento sobre remedios caseros para el problema de salud consultado y su utilización, presencia de conocimiento intergeneracional sobre métodos alternativos en salud, y existencia de conciencia de pérdida de las habilidades cuidadoras en la familia.

Con respecto al tamaño de la muestra, se entrevistaron a seis sujetos pertenecientes a dos secuencias familiares. La técnica de recogida de datos se realizó mediante la entrevista semiestructurada. Se realizó un análisis del discurso de tipo semántico desde un doble plano: intracasos e intercasos (Amezcua, 2002).

\section{RESULTADOS/DISCUSIÓN \\ Motivos de utilización de los servicios de urgencias}

Entre los motivos de utilización de los servicios de urgencias, destacan los problemas banales (catarro común y conjuntivitis) y los problemas urgentes (arritmias y cólico nefrítico):

Estos datos coinciden con los resultados de diversos estudios. En uno de ellos se ponía de manifiesto que entre los 15 diagnósticos más frecuentes en urgencias se encontraban: fiebre $(2,5 \%)$, rinofaringitis aguda/resfriado común $(2,4 \%)$, otitis media aguda $(2 \%)$, conjuntivitis aguda (1\%) (Palanca, 2010; Pérez, 2009).

En ambas secuencias vemos que es la generación más joven la que acude a urgencias por un problema de escasa gravedad, mientras que la generación más mayor lo hace por una dolencia de urgencia:

"Dolor en la garganta, (con tos) y en el oído en una primera visita, después volví a acudir porque me dio fiebre" (Serbal).

"Hace poco no, yo acudí hace unos tres años, con una arritmia que me dio y en total en mis 93 años de vida habré ido unas cinco veces, contando la arritmia, dos veces de operación de vesícula y las demás por los dolores de vesícula que me daban" (Sauce).

"Acudí hace poco, por dolores de mujer (con el tema del embarazo y tal), pero también fui después porque me dolía mucho la garganta" (Drosera).

"La última fue en noviembre, por una arritmia" (Draba).

Diversas investigaciones ponen de manifiesto que es la gente joven la que normalmente hace un uso inadecuado de estos servicios (hablan incluso de que la estimación del uso inapropiado de urgencias varía en un rango de $24-79 \%$ ). Ya que la personas mayores suelen acudir por problemas derivados de su patología crónica o debido a un cuadro de pluripatología (Palanca, 2010).

En relación a la utilización que hacen los informantes de los servicios de urgencia, aparecen tres motivaciones:

1. Los que acuden por iniciativa propia: "Dolor en la garganta, (con tos) y en el oído en una primera visita, después volví a acudir porque me dio fiebre" (Serbal).

"Acudí hace poco, por dolores de mujer (con el tema del embarazo y tal), pero también fui después porque me dolía mucho la garganta" (Drosera).

2. Los que van presionados por familiares: 
“La primera vez que me dio el dolor de vesícula yo me aguantaba, me tomé mi manzanilla y leche calentica y reposé, ya fui a las urgencias porque no tenía más remedio, casi que me obligaron mis hijos" (Sauce).

3. Y finalmente los que son derivados desde el centro de salud:

“Me sentí palpitaciones e intenté remediarlo con una tila pero no me tranquilizaba, fui al centro de salud y de ahí a urgencias" (Draba).

En contra de lo que se pudiera pensar, la mayoría de las personas acuden a estos servicios por criterio propio, en relación con una minoría que lo hace por consejo de un profesional (Pérez et al., 2009).

\section{Utilización de remedios caseros para el problema de salud consultado}

La mitad de la muestra es capaz de describir algún tipo de remedio para el problema de salud consultado. El resto, aunque no sepa algún remedio para esa dolencia en específico, narra alguna terapia para paliar los síntomas que esta conlleva. Los remedios más comunes en la primera secuencia generacional son:

- La manzanilla. Su uso más frecuente es para los problemas oculares y como tratamiento paliativo en el dolor derivado de los cálculos biliares:

"Mi madre me enseñó que uno de los usos de la manzanilla era ese, para los problemas en el ojo" (Salvia).

"Antes usábamos la manzanilla o hervíamos agua con sal gorda" (refiriéndose a la conjuntivitis) (Sauce).

“La primera vez que me dio el dolor de vesícula yo me aguantaba, me tomé mi manzanilla y leche calentica y reposé" (Sauce).
- Zumo de naranja o limón con miel. Su uso más común es para tratar los síntomas derivados de un catarro común, es decir, lo utilizan para aliviar la tos y suavizar la garganta:

"Para la garganta, zumo de naranja con miel y para la tos de la noche media cebolla en la mesita de noche" (Serbal).

"Para la tos yo he utilizado mucho la media cebolla en la mesita de noche y también agua hervida con azúcar y al baño maría un zumo de naranja o de limón con miel" (Salvia).

En varios estudios se muestran las propiedades de la manzanilla, en uno de ellos se obtuvo, que algunas de las propiedades de esta planta eran: antiinflamatoria ocular (conjuntivitis), relajante y ligeramente sedante (dolores sobre todo de índole gastrointestinal), antiespasmódica entre otros (Gómez et al., 2015). Con respecto a la naranja y limón se ha demostrado sus propiedades antivirales, antialérgicas y antiinflamatorias, apoyando la necesidad incluso de aumentar la cantidad de estos alimentos en nuestra dieta (Martínez et al., 2002). Esto unido a la miel, que desde 1513 se sabía de sus propiedades terapéuticas donde destaca "desflemar la garganta", explica el hecho de su utilización en patologías respiratorias (Charro, 1999).

En la segunda secuencia generacional los remedios más utilizados son agua hervida con jengibre, miel, limón y naranja. Este remedio suele utilizarse para paliar los síntomas del catarro común o gripe:

“Para el dolor de garganta me sé los típicos: hierves en agua un poco de jengibre y luego le echas un poco de miel, limón y naranja" (Drosera).

"Para el dolor de garganta, se hace una infusión con jengibre, limón, naranja y miel de romero. Se corta el jengibre en rodajas, lo 
pones a hervir y el caldo que te sale lo echas en un vaso, al que le pones naranja, limón y una cucharada de miel de romero" (Dentaria).

A las propiedades citadas anteriormente de la miel, el limón y la naranja se une las propiedades antiinflamatorias y analgésicas del jengibre (Ojewole, 2006).

Es decir, el criterio de los entrevistados sobre el uso de diversos remedios, coincide con el de los estudios citados, demostrándose de esta manera que son prácticas totalmente empíricas.

Nos encontramos que tanto en la secuencia primera como en la segunda son las generaciones mayores las que siguen utilizando los remedios que les transmitieron sus generaciones anteriores:

"Yo solo voy a urgencias en casos graves, a mí cuando me da dolor de barriga, me tomo mi manzanilla y aguanto con el dolor como pueda. Solo voy cuando estoy mala, muy mal. Yo no abuso de médicos, y yo los respeto pero yo me apaño con lo que siempre he hecho" (Sauce).

"Suelo ir a urgencias cuando veo que yo sola no puedo hacerme nada, cuando me duele la barriga o me da conjuntivitis (tengo muchos problemas en los ojos), intento yo sola lavármelos con manzanilla o aguasal" (Draba).

Esto se produce porque la capacidad de autocuidado se está debilitando debido a los efectos de una medicina paternalista, donde el usuario "más que acudir a curarse, acude a ser curado" (Juárez et al., 2014).

En la muestra se puede ver cómo el promotor de estas prácticas es la mujer, solamente en un caso ha sido el personal sanitario:

"Utilizo mucho la media cebolla para la tos del niño. La pediatra nos dijo que es casi mejor que el humidificador porque no servía para todos los tipos de tos" (Serbal).
Esto es así porque la mujer es vista desde la prehistoria como fuente de cuidados, participando en todo lo relacionado con la salud de una forma directa, afianzando de esta forma su papel dentro del seno familiar (López, 2010). Por otra parte, es imprescindible la participación del profesional sanitario, y su necesidad de actuación. Además, la Enfermería específicamente tiene que ser la encargada de realizar labores educativas de una forma integral e incrementar la utilización de estas técnicas (Rodríguez et al., 2006).

\section{Existencia de conocimiento intergeneracional}

Al analizarlo por generaciones, en la primera secuencia generacional nos encontramos que el miembro más joven, que acudió a urgencias por un resfriado con síntomas determinados, es capaz de describir cómo cree que su madre actuaba en su misma situación:

"Mi madre utilizaba más la naranja y también la leche caliente con miel, la miel es muy recurrente" (Serbal).

Si esto lo comparamos con los remedios que su madre conoce para este tipo de dolencia podemos observar que existe traspaso de este conocimiento a la generación posterior, pero se pierde información en el proceso, pues conoce más remedios que su hija:

"Para la tos yo he utilizado mucho la media cebolla en la mesita de noche y también agua hervida con azúcar y al baño maría un zumo de naranja o de limón con miel y para la fiebre paños de agua fría en las articulaciones" (Salvia).

Sin embargo, al preguntarle sobre la actuación de su abuela frente a ese problema, no narra ningún remedio, pero asegura que hacía uso de ellos y lo sigue 
haciendo. El miembro más mayor, en este caso la abuela, vemos que sabe multitud de remedios, para el problema por el cual la nieta acudió a urgencias:

"Para la tos: limón o leche con miel y también zumo de naranja con miel eso te alivia la tos, y te suaviza la garganta. Con eso es con lo que nos hemos curado siempre, porque ahora te dan tus pastillas pero antes con esto iba muy bien. Para la fiebre tus paños de agua fría en los huesos y reposo, no te daban otra cosa y se pasaba ¿eh?".

"Para el resfriado sigo partiendo mi cebolla y la pongo en la mesita de noche" (Sauce).

Si analizamos ese conocimiento para el problema de salud de Salvia, vemos que para esta dolencia cree que su madre actuaba así:

"Mi madre ponía agua hervida con sal o manzanilla cuando mis hermanos tenían legañas o los ojos pegados" (Salvia).

Al preguntarle sobre su abuela, nos responde que no la conoció pero afirma que le consta que ella utilizaba muchos más remedios.

Si vemos lo que su madre responde cuando le preguntamos sobre qué utilizaba para este tipo de dolencia se observa que los remedios empleados coinciden con los que narra su hija:

"Antes usábamos la manzanilla o hervíamos agua con sal gorda. Es lo que nos han dado toda la vida nuestras madres" (Sauce).

Observamos que existe conocimiento intergeneracional para un mismo tipo de dolencia, pero a medida que las generaciones avanzan, se ha perdido parte de esa información. También se puede ver cómo este tipo de remedios han estado presentes en su cotidianidad siempre desde dentro del hogar, algo que en las generaciones presentes se está perdiendo.
Algunos estudios justifican esta pérdida o invisibilidad de estos saberes con el protagonismo que ha tomado en los últimos tiempos el sistema sanitario, que con una mirada incompleta de la realidad hace que la autonomía, el poder y las habilidades de los grupos humanos se vayan debilitando (López, 2010; Osorio et al., 2008).

Si analizamos la segunda secuencia generacional nos encontramos que para el resfriado común que es la patología por la cual Drosera acudió a urgencias, su padre en este caso cree que actuaba haciendo uso de leche y la miel, al igual que su madre:

"Mi madre sí que ha utilizado, leche con miel y mi padre también aunque menos, pero suelen ir al médico" (Drosera).

$\mathrm{Al}$ comprobar lo anterior con la respuesta del padre, vemos que apenas hay coincidencias:

"Para el dolor de garganta, se hace una infusión con jengibre, limón, naranja y miel de romero. Se corta el jengibre en rodajas, lo pones a hervir y el caldo que te sale lo echas en un vaso, al que le pones naranja, limón y una cucharada de miel de romero" (Dentaria).

Cuando le preguntamos sobre la actuación de su abuela responde cómo cada vez hace un uso menor de estos remedios, aunque sigue utilizando algunos de ellos. No es capaz de detallar ningún remedio para esa dolencia en específico, pero afirma que su abuela hacia más uso de esta medicina. Sin embargo, su abuela sí es capaz de narrar algún remedio para el problema por el cual su nieta acudió a los servicios de urgencias:

"Antes se utilizaba mucho la miel pura, con leche caliente o bien con zumo de limón. Cuando a las mujeres nos dolía el periodo, mi madre me daba (aún siendo muy pequeña) una copa de ginebra y menta" (Draba).

En esta secuencia generacional, a 
diferencia de la anterior, se puede ver cómo este conocimiento intergeneracional se ha visto más afectado conforme el tiempo transcurre, además al introducir la figura del padre, este traspaso de conocimiento se ve afectado ya que es la figura de la madre la que "ha mantenido la previsión que impone la responsabilidad sobre el cuidado de otras personas, esto la cultura masculina lo ha despreciado" (Antonio, 2012).

\section{Conciencia del proceso de pérdida de las habilidades cuidadoras}

El total de la muestra es consciente del proceso de pérdida de estas habilidades cuidadoras, coincidiendo en que cada vez se conservan menos conforme avanzan las generaciones.

Al estudiar los motivos por los cuáles piensan que este proceso se ha producido vemos que los predominantes en ambas secuencias generacionales son:

- Influencia de la farmacia moderna:

“También juegan un papel fundamental las farmacias, ya no hay persona que no tenga botiquín completo y para viajar lo primero que echas es la medicación, aunque no la necesites. Es curioso cómo nos hemos vuelto dependientes de las pastillas, y pienso que tiene mucho que ver con la publicidad, y la sobrecarga de información que nos mandan. Estás por ejemplo viendo la película y cada diez anuncios cuatro son de pastillas" (Salvia).

"Hoy en día es más factible tener un medicamento, $y$ en base a eso las empresas farmacéuticas tienen que hacer su negocio, y a ellos les interesa más tener que vendernos sus productos a que nosotros hagamos los nuestros, ellos te van a mandar un medicamento antes que remedios" (Dentaria).

- Pasividad por parte de la población:

"Además ir a urgencias es muy asequible, nos hemos vuelto muy cómodos en ese sentido porque lo máximo que te puede pasar es esperar en vez de media hora 5 horas" (Salvia).

"Ha habido mucho cambio desde que yo me acuerdo con mi madre, cuando hacía sus remedios a ahora, incluso yo misma a mis hijos no les he pasado apenas remedios será porque ya hay otros medios. Muchas de las cosas que hacía mi madre conmigo yo ya no lo he hecho también por comodidad "(Draba).

- Falta de confianza en estos remedios:

"Ahora no le prestamos tanta atención a estas cosas. Sobre todo en la juventud hay una falta de interés por este tipo de remedios y no le hacemos caso ni siquiera. Cuando se trata a lo mejor de cosmética como quitarte los granos o para el pelo sí que te puedes buscar la vida, mirando por internet remedios caseros como la camomila o el aloe vera, pero cuando la salud está de por medio nos da más miedo" (Drosera).

“Pero la gente ya no confía en eso, se piensa que no es efectivo y prefieren ir al médico. Mi hija que ya es más moderna que yo, enseguida que se pone mala, va a la consulta, y eso tampoco es asi" (Draba).

- Influencia de la medicina occidental:

"Pienso que la gente joven tiene otras fuentes de información, los medios de comunicación influyen mucho al igual que las redes sociales y entonces se dejan mucho llevar por esto y por la gente más profesional que ya tienen otra mentalidad y otros criterios que también lo van transmitiendo" (Salvia).

"El médico no sabe de remedios antiguos y tampoco te va a decir que los uses teniendo pastillas" (Sauce).

Diversos estudios apoyan los resultados anteriores explicados por los cambios 


\section{Cultura de los Cuidados}

culturales que suceden actualmente como la "medicalización de lo cotidiano" que vuelve pasivos a los ciudadanos. Afirman incluso que "en nuestra sociedad preferimos tener una enfermedad antes que una limitación" (Quintana, 2013). Evidentemente el sistema sanitario no queda exento de estos cambios, y el abuso de los servicios de urgencias puede explicarse por la falta de responsabilidad de los ciudadanos hacia su salud, así como la carencia de autocuidados. Por lo tanto, el proceso de pérdida de estos saberes puede justificarse con los cambios que actualmente acontecen: la comodidad de la sociedad guiada por una cultura de la inmediatez y la consecuencia de perder la responsabilidad de nuestra salud.

\section{BIBLIOGRAFÍA}

- Amezcua, M., Gálvez Toro, A. (2002). Los modos de análisis en investigación cualitativa en salud: perspectiva crítica y reflexiones en voz alta. Revista Española de Salud Pública, 5(76), 423-436.

- Antonio, N.B. (2012). Saberes con rostros de mujeres indígenas, conocimientos tradicionales y actividades agrícolas productivas, en el Ejido el Remolino, municipio de Huitiupán, Chiapas (Tesis de grado para optar por el título en Licenciatura en Desarrollo Sustentable). Chiapas: Universidad Intercultural de Chiapas. Recuperado de http://cuencagrijalva.ecosur.mx/cuenca_grijalva/p dfs/publicaciones/tesis/SP01 Tesis03 Nancy Beatr iz_Antonio_Miguel_Saberes_con_Rostros_de_Muj eres indigenas 032012.pdf.

- Charro Gorgojo, M.A. (1999). Tras la senda de la miel. Revista de Folklore, 227. Recuperado de http://www.funjdiaz.net/folklore/07ficha.cfm?id=1 $\underline{824}$.

- Echevarría Pérez, P. (2008). Un análisis antropológico de los patrones de uso y perfil del usuario de Terapias complementarias orientales. Gazeta de Antropología, 24, 24-28. Recuperado de http://www.ugr.es/ pwlac/G24 27Paloma Echeva rria_Perez.html.

- Glittenberg, J. (2010). Construcción de cuidados enfermeros culturalmente competentes. Index de Enfermería, 19(2-3), 134-136. Recuperado de http://scielo.isciii.es/scielo.php?script=sci_arttext\& pid=S1132-12962010000200013.

- González Romero, G.M. (2010). Saturación de las Urgencias Hospitalarias. Un análisis descriptivo de motivos. REDUCA (Enfermería, Fisioterapia $y$ Podología), 2(1). Recuperado de: http://revistareduca.es/index.php/reducaenfermeria/article/view/208.

- Gómez Ugarte, M., Reyes Rojas, S., Paredes Choque, L. (2015). La manzanilla y sus propiedades medicinales. Revista de Investigación e Información en Salud, 10 (54), 54-58. Recuperado de: http://www.revistasbolivianas.org.bo/scielo.php?p id=S2075-61942015000100008\&script=sci_abstract.

- Güell, O. (2008). Vamos a urgencias urja o no. El País [online]. Recuperado de http://elpais.com/diario/2008/04/03/sociedad/12071 73601_850215.html.

- Juárez Jiménez, M.V., Baena Bravo, A.J. (2014). El paradigma de las patologías banales y las urgencias. Carta al director. Medicina de familia Andalucía, 15(2), 70-71. Recuperado de http://www.samfyc.es/Revista/PDF/v15n2/v15n2 11 carta.pdf.

- López, L. (2010). Los saberes culturales en un mundo globalizado. Index de Enfermería, 19(2-3), 8182.

- Martínez Flórez, S., González Gallego, J., Culebras, J.M. (2002). Los flavonoides: propiedades y acciones antioxidantes. Nutrición Hospitalaria, 17(6), 271-278.

- Ministerio de Sanidad, Servicios Sociales e Igualdad. Barómetro Sanitario (2015). Recuperado de:

http://pestadistico.inteligenciadegestion.msssi.es/p ublicoSNS/comun/ArbolNodos.aspx.

- Miró, O. (2010). ¿Hacia dónde caminamos?: Urgencias 2030. Anales del Sistema Sanitario de Navarra, 33, 229-235. Recuperado de http://scielo.isciii.es/scielo.php?pid=S113766272010000200022\&script=sci_arttext.

- Ojewole, J.A. (2006). Analgesic, antiinflammatory and hypoglycaemic effects of ethanol extract of Zingiber officinale (Roscoe) rhizomes (Zingiberaceae) in mice and rats. Phytotherapy Research, 20(9), 764-772. Recuperado de http://onlinelibrary.wiley.com/doi/10.1002/ptr.195 2/abstract.

- Osorio M., López, L. (2008). Competencia cultural en salud: necesidad emergente en un mundo globalizado. Index de Enfermería, 17(4), 266-270. Recuperado de http://scielo.isciii.es/scielo.php?pid=S1132$\underline{12962008000400010 \& \text { script=sci_arttext. }}$

- Palanca Sánchez, I., Eola Somozan, J., Mejía 
Estebaranz, F., (2010). Unidad de urgencias hospitalarias: Estándares y recomendaciones. Informes, estudios e investigación. Madrid.

- Ministerio de Sanidad, Servicios Sociales e Igualdad. Recuperado de http://www.msssi.gob.es/organizacion/sns/planCa lidadSNS/docs/UUH.pdf.

- Pérez Ciordia, I., Guillén Grima, F. (2009). Urgencias hospitalarias y extrahospitalarias en Navarra: Razones que las motivan. Anales del Sistema Sanitario de Navarra, 32 (3), 371-384.

- Quintana Gómez, J.L. (2013). La tentación de tratar enfermedades banales. Actualización en medicina de familia, 9(4),182-183. Recuperado de http://amfsemfyc.com/web/article ver.php?id=1127.
- Rodríguez Domínguez, I., Santana Gutiérrez, O., Recio López, O., Fuentes Naranjo, M. (2006). Beneficios del Aloe vera 1. (sábila) en las afecciones de la piel. Revista Cubana de Enfermería, 22(3). Recuperado de http://bvs.sld.cu/revistas/enf/vol22_3_06/enf04306r htm.

- Trinidade Santos, E.M.D., Ángel Vargas, L., Chad Pellón, L.H. (2013). La Interculturalidad en el mundo globalizado y sus impactos en el proceso salud-enfermedad: contribuciones de la Enfermería. Index de Enfermería, 22(4), 214-218. Recuperado de http://scielo.isciii.es/scielo.php?pid=S1132$\underline{12962013000300005 \& \text { script }=\text { sci arttext. }}$. 